\title{
An Optimal Scaling Scheme for DCO-OFDM Based Visible Light Communications
}

\author{
Rui Jiang*, Qi Wang, Fang Wang, Linglong Dai, Zhaocheng Wang \\ Tsinghua National Laboratory for Information Science and Technology (TNList), \\ Department of Electronic Engineering, Tsinghua University, Beijing 100084, China
}

\begin{abstract}
DC-biased optical orthogonal frequency-division multiplexing (DCO-OFDM) is widely used in visible light communication (VLC) systems to provide high data rate transmission. As intensity modulation with direct detection (IM/DD) is employed to modulate the OFDM signal, scale up the amplitude of the signal can increase the effective transmitted electrical power whereas more signals are likely to be clipped due to the limited dynamic range of LEDs, resulting in severe clipping distortion. Thus, it is crucial to scale the signal to find a tradeoff between the effective electrical power and the clipping distortion. In this paper, an optimal scaling scheme is proposed to maximize the received signal-to-noise-plus-distortion ratio (SNDR) with the constraint of the radiated optical power in a practical scenario where DC bias is fixed for a desired dimming level. Simulation results show that the system with the optimal scaling factor outperforms that with fixed scaling
\end{abstract}

${ }^{*}$ Corresponding author

Email addresses: jr14@mails.tsinghua.edu.cn (Rui Jiang), qiwang11@mails.tsinghua. edu.cn (Qi Wang), fang-wang10@mails.tsinghua.edu.cn (Fang Wang), daill@tsinghua.edu.cn (Linglong Dai), zcwang@tsinghua.edu.cn (Zhaocheng Wang) 
factor under different equivalent noise power in terms of the bit error ratio (BER) performance.

Keywords: Visible light communication, DCO-OFDM, scaling factor, clipping distortion, dimming control.

\section{Introduction}

Scarcity of radio frequency $(\mathrm{RF})$ spectrum is an increasingly prominent issue in wireless communication as the mobile data grows explosively. Visible light communication (VLC) can be applied to address the problem since the bandwidth of the unregulated visible light spectrum can be up to terahertz [1-4]. In VLC systems, intensity modulation with direct detection (IM/DD) is employed to modulate the signal, whereby the instantaneous optical power of the transmitter is proportional to the intensity/amplitude of the electrical signal [5]. As LEDs have a maximum permissible current and a turn-on voltage which corresponds to a minimum permissible current, the signal exceeding this dynamic range would be clipped and suffers from

clipping distortion. Several researches on the clipping distortion modeling have been done in [6-8]. In the IM/DD-based VLC systems, scaling up the amplitude of the signal can increase the effective transmitted electrical power while severe clipping distortion is imposed, since more signals might be clipped. Therefore, proper level scaling of the signal contributes to better system performance.

Orthogonal frequency-division multiplexing (OFDM) is widely used in IM/DD-based VLC systems to provide high data rate transmission [9-13]. DC bias is added to the OFDM signal to make it non-negative. This modified 


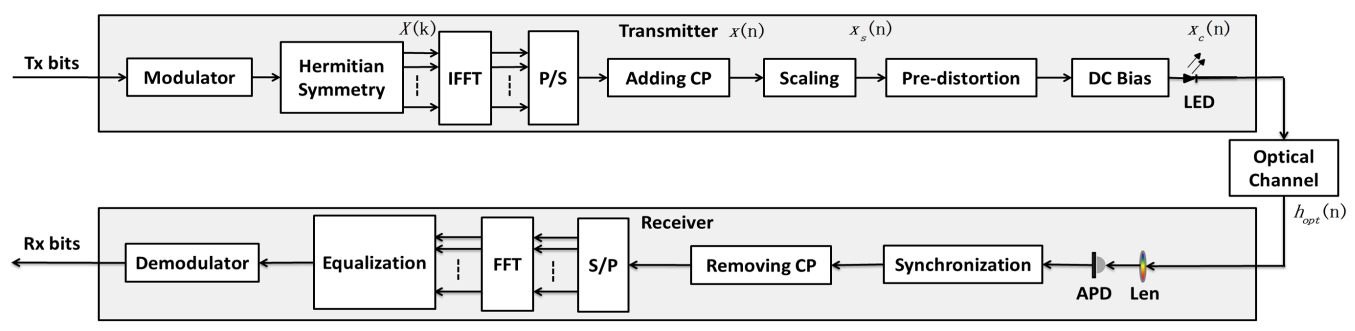

Fig. 1. Block diagram of DCO-OFDM VLC system.

OFDM scheme is called DCO-OFDM. In most existing work, an optimal DC bias is considered to improve the system performance [14, 15]. However, in a practical scenario where users adjust the dimming level through analog dimming, DC bias is set to a specific value [16]. To the best of our knowledge, this situation has not been considered in the existing work.

In this paper, an optimal scaling scheme under a fixed DC bias is proposed to find a tradeoff between the effective transmitted electrical power and the clipping distortion. The optimization problem is modeled as maximizing the effective received signal-to-noise-plus-distortion ratio (SNDR) with the constraint of the optical power. Simulation results show that the optimal scaling scheme can significantly improve the system performance.

\section{System Model}

The block diagram of the DCO-OFDM VLC system is illustrated in Fig. 1. The time-domain OFDM signal $x(n)$ is obtained by inverse fast Fourier transform (IFFT) from the modulated complex symbols $X(k)$. As the transmitted signal must be real-valued for IM/DD systems, $X(k)$ follows Hermitian sym- 
metry as

$$
X(k)=X^{*}(N-k), k=0,1, \ldots, N / 2-1,
$$

where the subcarriers $X(0)$ and $X(N / 2)$ are set to zero. A cyclic prefix (CP) is added in front of each OFDM symbol to avoid the inter-symbol interference (ISI). The time-domain signal $x(n)$ is then scaled with a scaling factor $\alpha$. For clarity, the scaled signal is denoted as $x_{s}(n)$. It should be noted that to overcome the adverse effect of the nonlinear transfer characteristics of the LEDs, pre-distortion can be applied to obtain a linear dynamic range between the maximum permissible current and the minimum permissible current [17]. Subsequently, a DC bias is added to make the pre-distorted signal non-negative. However, since the dynamic range is limited, the pre-distorted signal input into LEDs might be clipped and suffers from the undesired clipping distortion. Through IM/DD modulation, the clipped signal $x_{c}(n)$ is converted to the optical signal for emission.

At the receiver, the optical signal is collected by the hemisphere lens. Photodiode (PD) or avalanche photodiode (APD) is used as the detector. The shot noise, the receiver preamplifier noise and ambient light are modeled as additive white Gaussian noise (AWGN) [5]. Thus, the received signal can be expressed as

$$
y(n)=x_{c}(n) * h_{o p t}(n)+n_{g}(n)
$$

where $h_{\text {opt }}(n)$ and $n_{g}(n)$ denote the discrete forms of the channel impulse response $h_{\text {opt }}(t)$ and AWGN. Fast Fourier transform (FFT) is performed to recover the symbol from the sampled data. After one-tap equalization, the frequency symbols are demodulated. 


\section{Optimal Scaling Scheme}

According to central limit theorem, if the number of the subcarriers $N$ is large enough, the time-domain signal $x(n)$ follows a Gaussian distribution, i.e. $x(n) \sim N\left(0, \sigma_{x}^{2}\right)[6]$. The scaled signal can be written as $x_{s}(n)=\alpha x(n)$, and it also follows a Gaussian distribution as $x_{s}(n) \sim N\left(0, \alpha^{2} \sigma_{x}^{2}\right)$. The electrical power of the scaled signal is given by

$$
P_{x_{s}}=E\left(x_{s}^{2}\right)=\alpha^{2} \sigma_{x}^{2}
$$

After pre-distortion, the equivalent transfer characteristics of the LEDs can be expressed as [14]

$$
\varphi\left(x_{s}\right)= \begin{cases}\xi_{\min }, & \text { if } x_{s} \leqslant \xi_{\min } \\ x_{s}, & \text { if } \xi_{\min }<x_{s}<\xi_{\max } \\ \xi_{\max }, & \text { if } x_{s} \geqslant \xi_{\max },\end{cases}
$$

where $\xi_{\min }, \xi_{\max }$ are the minimum and maximum permissible signal amplitude. As DC bias $\xi_{D C}$ only affects the unused 0th subcarrier, the equivalent signal is considered in the following analysis with DC bias shifted to zero. Consequently, we set $x_{\min }=\xi_{\min }-\xi_{D C}$ and $x_{\max }=\xi_{\max }-\xi_{D C}$. Correspondingly, the clipped signal is expressed as $x_{c}=\varphi\left(x_{s}\right)-\xi_{D C}$, and its probability density function $(\mathrm{PDF})$ is given by [6]

$$
p\left(x_{c}\right)= \begin{cases}{\left[1-Q\left(\frac{x_{\min }}{\alpha \sigma_{x}}\right)\right] \delta\left(x_{c}-x_{\min }\right),} & \text { if } x_{c}=x_{\min } \\ \frac{1}{\sqrt{2 \pi} \alpha \sigma_{x}} e^{-\frac{x_{c}^{2}}{2 \alpha^{2} \sigma_{x}^{2}},} & \text { if } x_{\min }<x_{c}<x_{\max } \\ Q\left(\frac{x_{\max }}{\alpha \sigma_{x}}\right) \delta\left(x_{c}-x_{\max }\right), & \text { if } x_{c}=x_{\max },\end{cases}
$$

where we have $Q(y)=\frac{1}{\sqrt{2 \pi}} \int_{y}^{+\infty} e^{-\frac{u^{2}}{2}} \mathrm{~d} u$ and $\delta(\cdot)$ is the dirac delta function. 
The electrical power of the transmitted clipped OFDM signal is defined as $[7,18]$

$$
P_{x_{c}}^{t}=P_{x_{c}}^{s}+P_{x_{c}}^{n}
$$

where $P_{x_{c}}^{n}$ is the electrical power caused by clipping distortion and $P_{x_{c}}^{s}$ is the effective transmitted electrical power, which is expressed as

$$
\begin{aligned}
P_{x_{c}}^{s} & =\frac{1}{2 \pi \alpha^{2} \sigma_{x}^{2}}\left[\int_{x_{\min }}^{x_{\max }} e^{-\frac{x_{s}^{2}}{2 \alpha^{2} \sigma_{x}^{2}}} \mathrm{~d} x\right]^{2} * P_{x_{s}} \\
& =\left[Q\left(\frac{x_{\min }}{\alpha \sigma_{x}}\right)-Q\left(\frac{x_{\max }}{\alpha \sigma_{x}}\right)\right]^{2} \alpha^{2} \sigma_{x}^{2} .
\end{aligned}
$$

Since DC bias may not be in the middle position of the dynamic range of LEDs, the upside and downside of the signal will suffer from different clipping levels. Thus, the mean of the clipped signal is nonzero. In this situation, it has an extra DC component $E\left(x_{c}\right)$, which is expressed as

$$
\begin{aligned}
E\left(x_{c}\right)= & \int_{x_{\min }}^{x_{\max }} x_{c} p\left(x_{c}\right) \mathrm{d} x_{c} \\
= & \frac{1}{\sqrt{2 \pi}}\left(e^{-\frac{x_{\min }^{2}}{2 \alpha^{2} \sigma_{x}^{2}}}-e^{-\frac{x_{\max }^{2}}{2 \alpha^{2} \sigma_{x}^{2}}}\right) \alpha \sigma_{x} \\
& +\left[1-Q\left(\frac{x_{\min }}{\alpha \sigma_{x}}\right)\right] x_{\min }+Q\left(\frac{x_{\max }}{\alpha \sigma_{x}}\right) x_{\max }
\end{aligned}
$$

However, at the receiver, it only falls onto the unused 0th subcarrier. As a result, the reduced electrical power in the clipping process $P_{x_{c}}^{r d}$ includes the power of the clipped part of the signal $P_{\text {clip }}$ and the power of the extra DC component $P_{D C}^{e x}$, which can be further expressed by (9). 


$$
\begin{aligned}
P_{x_{c}}^{r d}= & \overbrace{\left\{\int_{x_{\min }}^{x_{\max }} x_{c} p\left(x_{c}\right) \mathrm{d} x_{c}\right\}^{2}}^{P_{D C}^{e x}} \overbrace{}^{P_{c l i p}} \overbrace{-\infty}^{x_{\min }}\left(x_{s}^{2}-x_{\min }^{2}\right) p\left(x_{s}\right) \mathrm{d} x_{s}+\int_{x_{\max }}^{+\infty}\left(x_{s}^{2}-x_{\max }^{2}\right) p\left(x_{s}\right) \mathrm{d} x_{s} \\
= & {\left[1-Q\left(\frac{x_{\min }}{\alpha \sigma_{x}}\right)+Q\left(\frac{x_{\max }}{\alpha \sigma_{x}}\right)+\frac{1}{2 \pi}\left(e^{\left.\left.-\frac{x_{\min }^{2}}{2 \alpha^{2} \sigma_{x}^{2}}-e^{-\frac{x_{\max }^{2}}{2 \alpha^{2} \sigma_{x}^{2}}}\right)^{2}\right] \alpha^{2} \sigma_{x}^{2}}\right.\right.} \\
& -\left[Q\left(\frac{x_{\min }}{\alpha \sigma_{x}}\right)-Q\left(\frac{x_{\min }}{\alpha \sigma_{x}}\right)^{2}\right] x_{\min }^{2}-\left[Q\left(\frac{x_{\max }}{\alpha \sigma_{x}}\right)-Q\left(\frac{x_{\max }}{\alpha \sigma_{x}}\right)^{2}\right] x_{\max }^{2} \\
& +\left\{\sqrt{\frac{2}{\pi}}\left(e^{-\frac{x_{\min }^{2}}{2 \alpha^{2} \sigma_{x}^{2}}}-e^{-\frac{x_{\max }^{2}}{2 \alpha^{2} \sigma_{x}^{2}}}\right)\left[1-Q\left(\frac{x_{\min }}{\alpha \sigma_{x}}\right)\right]-\frac{1}{\sqrt{2 \pi}} e^{-\frac{x_{\min }^{2}}{2 \alpha^{2} \sigma_{x}^{2}}}\right\} \alpha \sigma_{x} x_{\min } \\
+ & 2\left[1-Q\left(\frac{x_{\min }}{\alpha \sigma_{x}}\right)\right] Q\left(\frac{x_{\max }}{\alpha \sigma_{x}}\right) x_{\min } x_{\max }
\end{aligned}
$$

Then the power of the clipping distortion is given by [7]

$$
P_{x_{c}}^{n}=P_{x_{s}}-P_{x_{c}}^{r d}-P_{x_{c}}^{s}
$$

and the received electrical signal power is expressed as

$$
P_{x_{c}}^{r}=\left|H_{o p t}(f)\right|^{2} P_{x_{c}}^{t}=\left|H_{o p t}(f)\right|^{2} P_{x_{c}}^{s}+\left|H_{o p t}(f)\right|^{2} P_{x_{c}}^{n}
$$

where $H_{\text {opt }}(f)$ is the frequency response of the optical channel with line of sight (LOS), which is the Fourier transform of $h_{\text {opt }}(t)$ and can be given by [5]

$$
H_{\text {opt }}(f)= \begin{cases}\frac{A}{d^{2}} R(\phi) T(\theta) g(\theta) \cos (\theta), & \text { if } 0 \leqslant \theta \leqslant \Theta_{c} \\ 0, & \text { if } \theta>\Theta_{c}\end{cases}
$$


where $A$ is the detector area, $d$ is the distance between the transmitter and the receiver, $\theta$ is the angle of incidence, $R(\phi)$ is the transmitter radiant intensity, $T(\theta)$ is the signal transmission of the filter, $g(\theta)$ is the concentrator gain and $\Theta_{c}$ is the field of view (FOV) of the concentrator. Thus, the received SNDR is defined as

$$
\begin{aligned}
\mathrm{SNDR} & =\frac{\left|H_{o p t}(f)\right|^{2} P_{x_{c}}^{s}}{\left|H_{o p t}(f)\right|^{2} P_{x_{c}}^{n}+N_{g}} \\
& =\frac{P_{x_{c}}^{s}}{P_{x_{s}}-P_{x_{c}}^{r d}-P_{x_{c}}^{s}+\frac{N_{g}}{\left|H_{o p t}(f)\right|^{2}}},
\end{aligned}
$$

where $N_{g}$ is the noise power. Then the bit error ratio (BER) in M-QAM OFDM-based VLC systems can be approximately expressed as [19]

$$
\begin{aligned}
P_{b}= & \frac{2(\sqrt{M}-1)}{\sqrt{M} \log 2(\sqrt{M})} Q\left(\sqrt{\frac{3}{M-1} \mathrm{SNDR}}\right) \\
& +\frac{2(\sqrt{M}-2)}{\sqrt{M} \log 2(\sqrt{M})} Q\left(3 \sqrt{\frac{3}{M-1} \mathrm{SNDR}}\right) .
\end{aligned}
$$

Therefore, in order to achieve the best BER performance, the optimal scaling scheme is described as maximizing the received SNDR, which is formulated as

$$
\begin{aligned}
& \max _{\alpha} \operatorname{SNDR}=\frac{P_{x_{c}}^{s}}{P_{x_{s}}-P_{x_{c}}^{r d}-P_{x_{c}}^{s}+\frac{N_{g}}{\left|H_{o p t}(f)\right|^{2}}}, \\
& \text { s.t. : } \\
& \qquad \alpha>0, \\
& \quad P_{o p t, \min } \leqslant P_{o p t}^{t} \leqslant P_{o p t, \max },
\end{aligned}
$$

where $P_{o p t}^{t}$ is the radiated optical power and expressed as $P_{o p t}^{t}=E\left(x_{c}\right)+\xi_{D C}$. For illumination requirement, the optical power should be in the defined 
range including $\xi_{D C}$. The maximum and minimum optical signal power allowed is $P_{o p t, \max }$ and $P_{o p t, \text { min }}$. As $\left|H_{o p t}(f)\right|$ is proportional to $d^{-2}$, the received SNDR at different positions is different. To compare the received SNDR at different positions, the equivalent noise power is defined by $\frac{N_{g}}{\left|H_{o p t}(f)\right|^{2}}$. $P_{x_{s}}$, $P_{x_{c}}^{s}$ and $P_{x_{c}}^{r d}$ can be substituted by (3), (7) and (9).

To solve the above optimization problem, the characteristics of $E\left(x_{c}\right)$ should be considered. $E\left(x_{c}\right)$ is nonzero when the downside and upside of the signals suffer from different clipping levels. If $\xi_{D C}$ is above the middle position of the dynamic range (i.e. $x_{\min }+x_{\max }<0$ ), we have $\frac{\partial E\left(x_{c}\right)}{\partial \alpha}<0$. Thus, $E\left(x_{c}\right)$ is negative and decreases monotonically with respect to the scaling factor $\alpha$. On the contrary, it is positive and increases monotonically when $\xi_{D C}$ is below the middle position (i.e. $x_{\min }+x_{\max }>0$ ). Therefore, in (17), either $P_{o p t, \min }$ or $P_{o p t, \max }$ will determine the maximal scaling factor $\alpha_{\max }$. Then the feasible set can be expressed as $\left(0, \alpha_{\max }\right]$. Moreover, as the second order derivation of SNDR is non-positive, the optimization problem is concave. Therefore, the optimal scaling factor can be obtained by

$$
\alpha_{\text {opt }}= \begin{cases}\alpha^{*}, & \text { if the condition } C_{1} \text { is satisfied; } \\ \alpha_{\max }, & \text { otherwise }\end{cases}
$$

where the condition $C_{1}$ is defined as

$$
\frac{\partial \mathrm{SNDR}}{\partial \alpha^{*}}=0, \quad \exists \alpha^{*} \in\left(0, \alpha_{\max }\right]
$$

However, it is not simple to derive a closed-form solution for the optimal scaling factor. In our simulation, Newton method is used to obtain a numerical solution. 


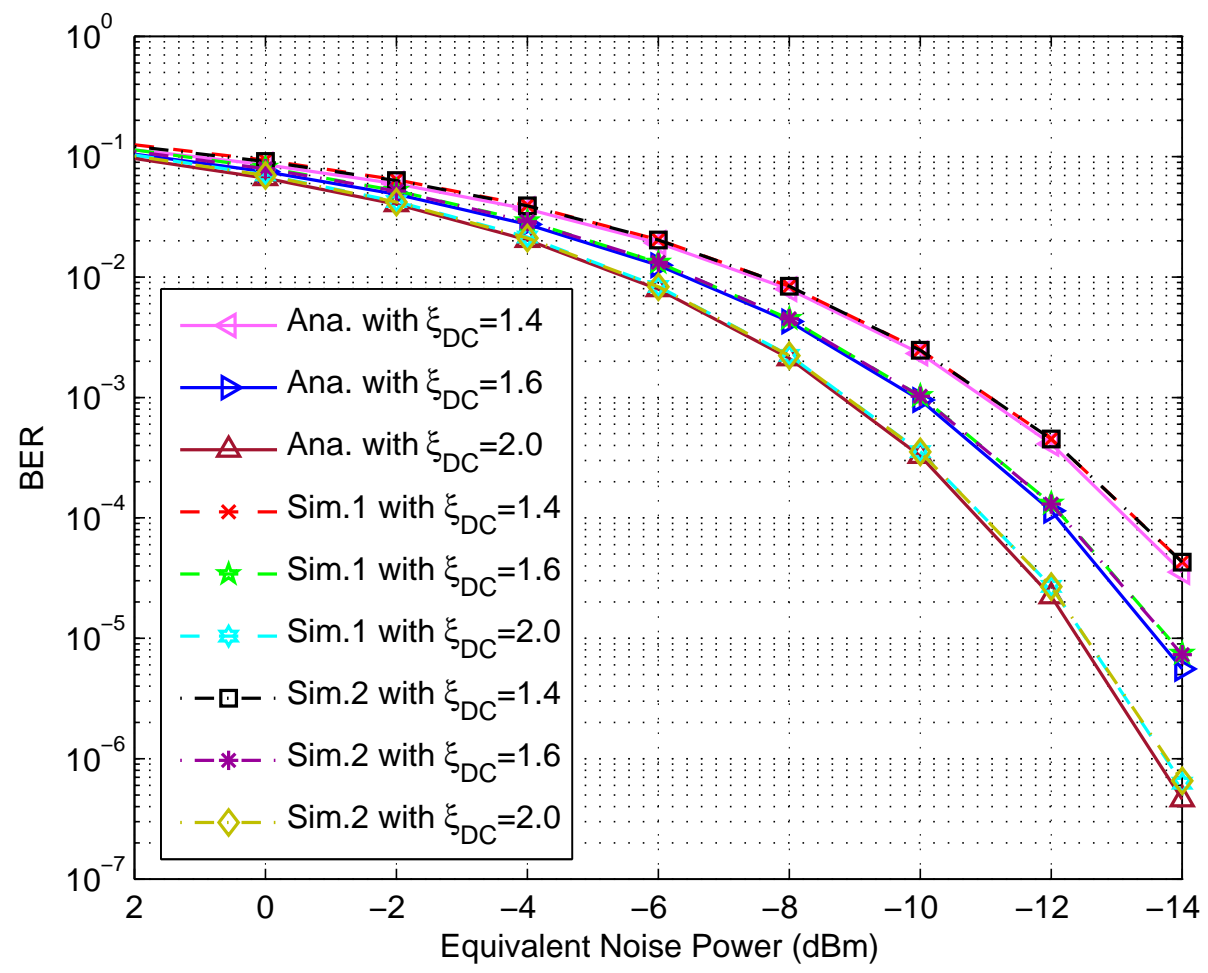

Fig. 2. BER perfomance of theoretical analysis and simulation for different DC bias values with 16QAM and 1024 subcarriers.

\section{Numerical Results}

In this section, simulation results of the optimal scaling factor are discussed. In the simulation, 16QAM with 1024 subcarriers are adopted. The electrical power of the OFDM signal without scaling is set as $10 \mathrm{dBm}$, which is normalized to be unit. The normalized minimum permissible signal amplitude is 0 while the normalized maximum permissible signal amplitude is 4. Three normalized DC bias values are considered, which are 1.4, 1.6 and 2. The corresponding range of the normalized optical power under the con- 
straint of illumination is set as $[1.3,1.5],[1.5,1.7]$ and $[1.9,2.1]$ respectively.

The BER performance of theoretical analysis and simulation for the optimal scaling factor are compared under different DC bias values in Fig. 2. The theoretical optimal BER is calculated using (14) with the optimal scaling factor obtained from (18) under different equivalent noise power. Two cases of simulation are considered here. In the first case, optimal BER is obtained through simulation with the optimal scaling factor calculated from (18). While for the second case, optimal BER is the best one chosen from the simulated BER for all the eligible scaling factors. It can be seen in Fig. 2 that, for a fixed DC bias value, three BER curves have a close match, which implies the system can achieve the best BER performance with the theoretical optimal scaling factor calculated from (18). Besides, BER can be better when DC bias is approaching to the middle position of the dynamic range of LEDs. The reason is that when DC bias is closer to the middle position, larger dynamic range can be utilized to transmit the signal without clipping.

The effect of the clipping distortion and equivalent noise on the optimal scaling factor is analyzed in Fig. 3. It is shown that the scaling factor becomes smaller when the equivalent noise power decreases. This is because a smaller scaling factor can alleviate the clipping distortion, which is the priority to improve system performance when the power of the equivalent noise is relatively low. On the contrary, larger scaling factor to generate higher signal power is adopted to overcome the adverse effect imposed by larger equivalent noise at the cost of severer clipping distortion. Due to the constraint of the radiated optical power, the scaling level of the signal is restricted. Thus, the curve of the scaling factor for $\xi_{D C}=1.4$ and $\xi_{D C}=1.6$ keeps constant 


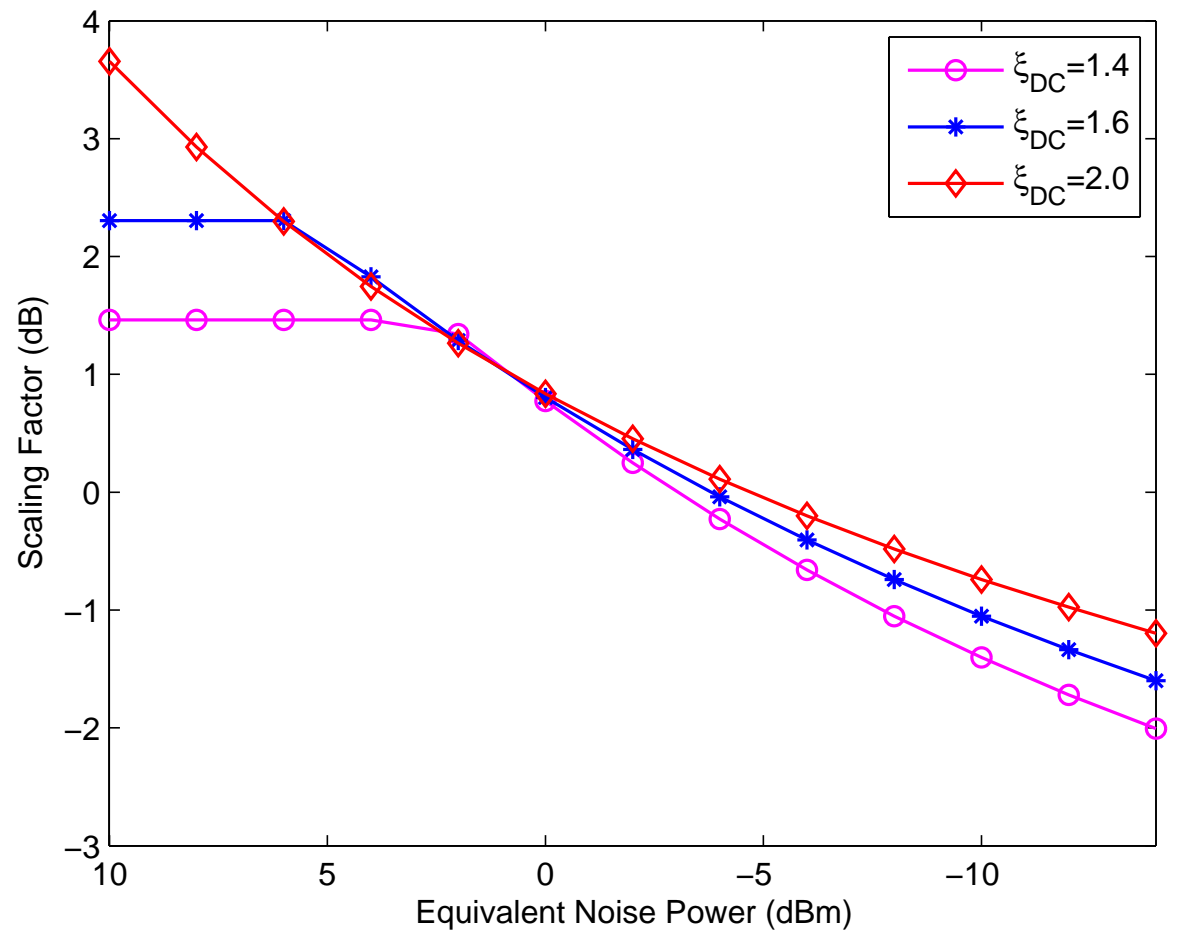

Fig. 3. Optimal scaling factor under different DC bias with 16QAM and 1024 subcarriers.

when the equivalent noise power is large. It also can be seen that when the equivalent noise power is small, the scaling factor is larger if the DC bias is closer to the middle position of the dynamic range since larger scope can be utilized.

As shown in Fig. 4, the BER performance of the fixed scaling factor and the optimal scaling factor is investigated. In the simulation for the fixed scaling factor, the scaling factor keeps constant under different equivalent noise power. The three fixed scaling factor values are set to $1.30 \mathrm{~dB},-0.04$ $\mathrm{dB}$ and $-0.74 \mathrm{~dB}$, respectively. The latter two values are the same as the 


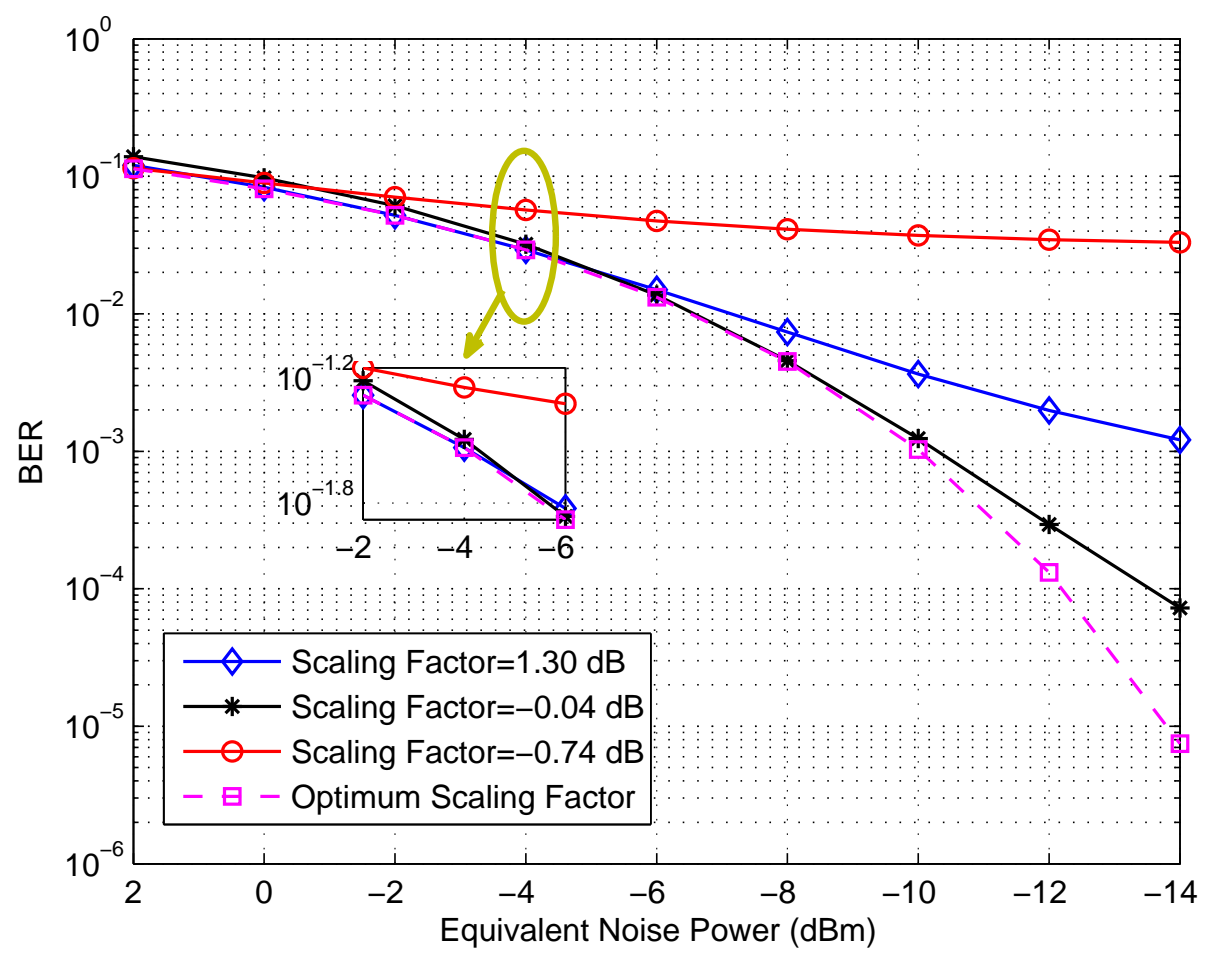

Fig. 4. BER performance comparison between the optimal scaling factor and the fixed scaling factor when $\xi_{D C}=1.6$ with 16QAM and 1024 subcarriers.

optimal scaling factor values when the equivalent noise power is $-4 \mathrm{dBm}$, $-8 \mathrm{dBm}$. Thus, each curve of the latter two fixed scaling factor values has an intersection point with the curve of the optimal scaling factor when the equivalent noise power is $-4 \mathrm{dBm},-8 \mathrm{dBm}$, as depicted in Fig. 4. The general trend is that the BER performance of the optimal scaling factor is the best in comparison with the fixed scaling factor under all equivalent noise power, and the performance is significantly improved, especially when the noise power is small. 


\section{Conclusions}

In this paper, an optimal scaling scheme is proposed for DCO-OFDM VLC systems, aiming at maximizing the received SNDR with the constraint of the radiated optical power. Simulation results show that there is a close match of BER performance between the theoretical analysis and the simulation for the optimal scaling factor. It can be concluded that the optimal scaling factor can significantly improve the system performance especially when equivalent noise power is relatively small. In addition, if DC bias is closer to the middle position of the dynamic range of LEDs, better BER performance can be achieved.

\section{References}

[1] A. Tsiatmas, C. P. M. J. Baggen, F. M. J. Willems, J.-P. M. G. Linnartz, J. W. M. Bergmans, An illumination perspective on visible light communications, IEEE Commun. Mag. 52 (7) (2014) 64-71.

[2] A. Jovicic, J. Li, T. Richardson, Visible light communication: opportunities, challenges and the path to market, IEEE Commun. Mag. 51 (12) (2013) 26-32.

[3] R. Zhang, J. Wang, Z. Wang, Z. Xu, C. Zhao, L. Hanzo, Visible light communications in heterogeneous networks: paving the way for usercentric design, IEEE Trans. Wireless Commun. 22 (2) (2015) 8-16.

[4] H. Li, Y. Zhang, X. Chen, C. Wu, J. Guo, Z. Gao, W. Pei, H. Chen, 682 Mbit/s phosphorescent white LED visible light communications utilizing 
analog equalized 16QAM-OFDM modulation without blue filter, Opt. Commun. 354 (2015) 107-111.

[5] J. M. Kahn, J. R. Barry, Wireless infrared communications, Proc. IEEE 85 (2) (1997) 265-298.

[6] S. Dimitrov, S. Sinanovic, H. Hass, Clipping noise in OFDMbased optical wireless communication systems, IEEE Trans. Commun. 60 (4) (2012) 1072-1081.

[7] S. Randel, F. Breyer, S. C. J. Lee, J. W. Walewski, Advanced modulation schemes for short-range optical communications, IEEE J. Sel. Topics Quantum Electron. 16 (5) (2010) 1280-1289.

[8] L. Chen, B. Krongold, J. Evans, Performance analysis for optical OFDM transmission in short-range IM/DD systems, J. Lightwave Technol. 30 (7) (2012) 974-983.

[9] J. Armstrong, OFDM for optical communications, J. Lightwave Technol. 27 (3) (2009) 189-204.

[10] Q. Wang, Z. Wang, L. Dai, Asymmetrical hybrid optical OFDM for visible light communications with dimming control, IEEE Photon. Technol. Lett. 27 (9) (2015) 974-977.

[11] H. Zhang, Y. Yuan, W. Xu, PAPR reduction for DCO-OFDM visible light communications via semi-definite relaxation, IEEE Photon. Technol. Lett. 26 (17) (2014) 1718-1721. 
[12] W. Xu, M. Wu, H. Zhang, X. You, C. Zhao, ACO-OFDM-specified recoverable upper clipping with efficient detection for optical wireless communications, IEEE Photon. J. 6 (5) (2014) 1-17.

[13] Q. Wang, Z. Wang, L. Dai, Iterative receiver for hybrid asymmetrically clipped optical OFDM, J. Lightwave Technol. 32 (22) (2014) 3869-3875.

[14] M. Zhang, Z. Zhang, An optimum DC-biasing for DCO-OFDM system, IEEE Commun. Lett. 18 (8) (2014) 1351-1354.

[15] R. Mesleh, H. Elgala, H. Hass, On the performance of different OFDM based optical wireless communication systems, J. Opt. Commun. Netw. 3 (8) (2011) 620-627.

[16] http://www.ti.com/lit/an/slyt084/slyt084.pdf

[17] H. Elgala, R. Mesleh, H. Haas, Non-linearity effects and predistortion in optical OFDM wireless transmission using LEDs, Inderscie. Int. J. Ultra Wideband Commun. Syst. 1(2) (2009) 143-150.

[18] R. Gross, D. Veeneman, SNR and spectral properties for a clipped DMT ADSL signal, in: Proc. IEEE International Conference on Communications, May 1994, pp. 843-847.

[19] K. Cho, D. Yoon, On the general BER expression of oneand two-dimensional amplitude modulations, IEEE Trans. Commun. 50 (7) (2002) 1074-1080. 